\title{
Synthesis of Cobalt-Iron Prussian Blue Analogues Nanotubes by CTAB Soft-Template Method
}

\author{
Peng Liu, ${ }^{\dagger}$ Chuanghui Liang, ${ }^{\dagger}$ Jianfeng Xu, ${ }^{\dagger}$ Jian Fang, ${ }^{\dagger}$ Jihua Zhao, ${ }^{\dagger, *}$ and Weiguo Shen ${ }^{\dagger, *}$ \\ ${ }^{\dagger}$ Department of Chemistry, Lanzhou University, Lanzhou 730000, China. E-mail: zhaojihua@lzu.edu.cn \\ ${ }^{\ddagger}$ Department of Chemistry, East China University of Science and Technology, Shanghai 200237, China \\ Received September 24, 2009, Accepted January 23, 2010
}

\begin{abstract}
Three cobalt-iron Prussian Blue Analogues (PBAs) nanotubes contained with different alkali metal cations of K, Rb or Cs, respectively, were prepared by using cetyltrimethylammonium bromide (CTAB)/ethanol-water micelles as soft templates. The products were characterized by energy-dispersive X-ray spectroscopy (EDS), X-ray diffraction (XRD), transmission electron microscopy (TEM) and scanning electron micrograph (SEM), which confirmed the composition of the substances and their unique nanotube structures. Furthermore, the formation mechanism of the PBAs nanotubes was discussed and provided useful insight for further synthesis of nanotubes of other Prussian blue analogues.
\end{abstract}

Key Words: Prussian blue analogues, Soft template method, CTAB, Nanotubes

\section{Introduction}

Transition metal hexacyanometalates with the general formula of $\mathrm{A}_{\mathrm{h}} \mathrm{M}_{\mathrm{k}}[\mathrm{N}(\mathrm{CN})]_{1} \cdot \mathrm{xH}_{2} \mathrm{O}(\mathrm{h}, \mathrm{k}, 1$, and $\mathrm{x}$ are stoichiometric numbers; $\mathrm{A}$ is an alkali metal cation; $\mathrm{M}$ and $\mathrm{N}$ are high-spin (HS) and low-spin (LS) divalent or trivalent transition metal ions, respectively.) present an important class of mixed-valence compounds, namely Prussian blue analogues (PBAs). ${ }^{1}$ With the alkali metal cation of $\mathrm{A}$, which provide charge compensation located in the tetrahedral sites, ${ }^{2}$ these compounds are inclined to crystallize in face-centered cubic (FCC) structures. If no alkali metal cations exist in there structures, however, water molecules will instead locate in these sites. In this case, the inherent structures become loose and the crystallization will be difficult. ${ }^{3}$

In recent years, there have been quite a few techniques developed for preparing such materials, for example, porous alumina, ${ }^{4}$ stearylamine, ${ }^{5}$ ionic liquids, ${ }^{6}$ mesostructured silica, ${ }^{7}$ sodium hexametaphosphate, ${ }^{8}$ apoferritin, ${ }^{9}$ polyvinylpyrrolidone, ${ }^{10}$ sol-gel, ${ }^{11,12,13}$ anodic aluminum oxide ${ }^{14}$ and microemulsion. ${ }^{15,16}$ Although considerable efforts have been put on the syntheses of PBAs materials, relatively few attempts ${ }^{17}$ have been on the produce of the tubular Prussian blue analogues, let alone the cobalt-iron PBAs nanotubes.

Most recently, our group ${ }^{15,16}$ obtained highly oriented cubic, hollow cubic, spherical nanoparticles and films of cobalt-iron PBAs via microemulsion technique. By utilizing similar methodology, herein we report an approach for the growth of nanotubes of PBAs using acetyltrimethylammonium bromide (CTAB)/ethanol-water micelles as soft templates. To the best of our knowledge, this is the first demonstration that the nanotubes of cobalt-iron PBAs are synthesized on the basis of the micelles method, with CTAB as the soft template.

\section{Experimental}

Materials. The cationic surfactant CTAB was purchased from Beijing Chemical Reagent Limited Company, and ethanol from
Tianjin Chemical Reagent Limited Company. Potassium ferricyanide $\left(\mathrm{K}_{3} \mathrm{Fe}(\mathrm{CN})_{6}\right)$, cobalt chloride hexahydrate $\left(\mathrm{CoCl}_{2} \cdot 6 \mathrm{H}_{2} \mathrm{O}\right)$ cesium chloride $(\mathrm{CsCl})$ and rubidium chloride $(\mathrm{RbCl})$ were purchased from Tianjin Guangfu Fine Reagent Factory. They are all analytical grade reagents. Deionized water was used throughout all the experiments.

Preparation of PBAs. Compound 1 was synthesized by mixing two types of solution together under magnetically stirring: one was a mixture of $1.17 \mathrm{~g} \mathrm{CTAB}, 10 \mathrm{~mL}$ ethanol and $10 \mathrm{~g}$ of a $1 \times 10^{-2} \mathrm{~mol} \cdot \mathrm{L}^{-1}$ solution of cobalt chloride hexahydrate, and the other was a mixture of $1.17 \mathrm{~g} \mathrm{CTAB}, 10 \mathrm{~mL}$ ethanol and $10 \mathrm{~g}$ of a $1 \times 10^{-2} \mathrm{~mol} \cdot \mathrm{L}^{-1}$ solution of potassium ferricyanide. The mixed solution continued to be magnetically stirred for $10 \mathrm{~h}$ at $25^{\circ} \mathrm{C}$. Thereafter, the reaction solutions were centrifuged at $12000 \mathrm{rpm}$ for about $2 \mathrm{~min}$. The precipitates were then thoroughly washed in a 1:1 (V/V) mixture of ethanol and water 7 times to remove the contaminated surfactant and other inorganic substances from the particles. Eventually, the products were dried under vacuum at $50{ }^{\circ} \mathrm{C}$.

To introduce other alkali cations into the structure, compounds $\mathbf{2}$ and $\mathbf{3}$ were both synthesized by mixing two micelle solutions, each of which was prepared from mixing $1.17 \mathrm{~g}$ $\mathrm{CTAB}$ and $10 \mathrm{~mL}$ ethanol with addition of $10 \mathrm{~g}$ of $0.03 \mathrm{M}$ $\mathrm{RbCl} / 0.01 \mathrm{M} \mathrm{CoCl}_{2}$ (compound 2) and 0.03 $\mathrm{M} \mathrm{CsCl} / 0.01 \mathrm{M}$ $\mathrm{CoCl}_{2}$ (compound 3 ) or $0.01 \mathrm{M} \mathrm{K}_{3} \mathrm{Fe}(\mathrm{CN})_{6}$ aqueous solution,

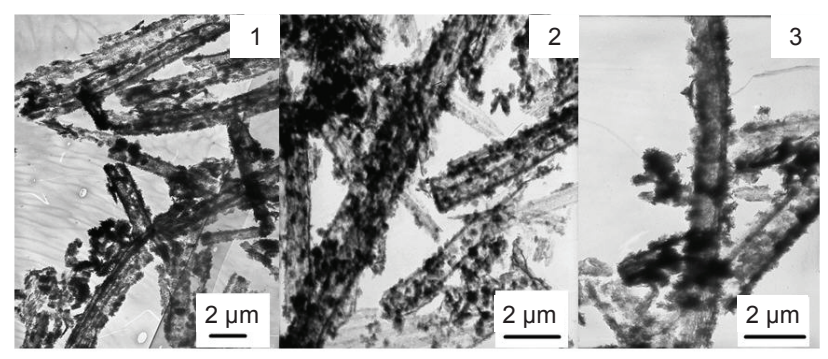

Figure 1. TEM images of PBAs nanotubes, (1) compound 1; (2) compound 2 ; (3) compound 3 . 


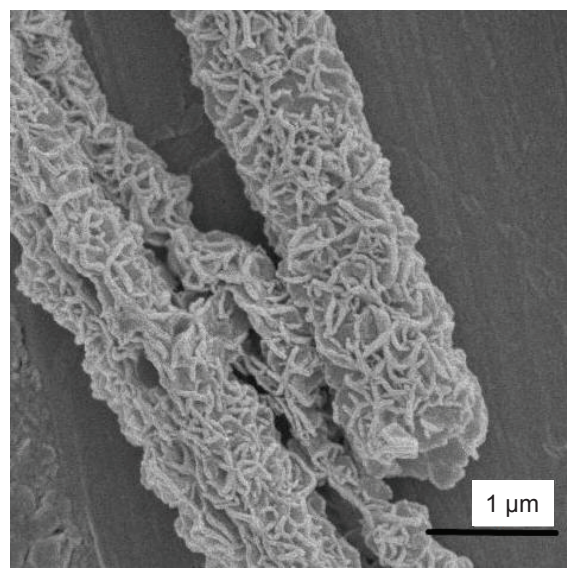

Figure 2. SEM image of the compound 1 nanotubes.

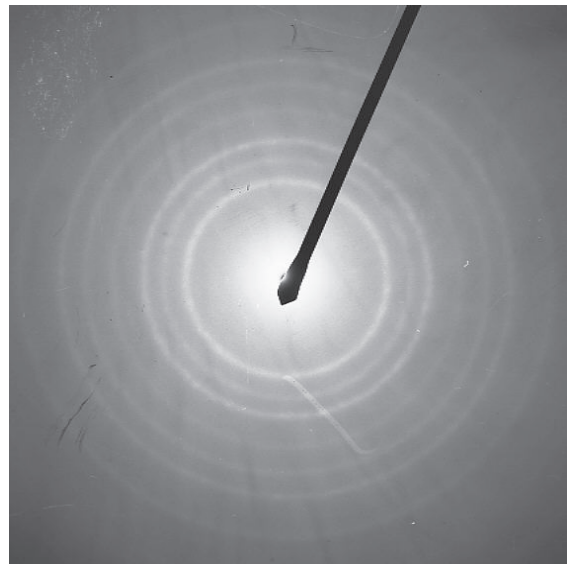

Figure 3. SAED pattern of the compound 1 nanotubes.

while other steps as those in the synthesis of compound $\mathbf{1}$ were unchanged.

Measurements. The powder X-ray diffraction (XRD) patterns $(\mathrm{Cu} \mathrm{K} \alpha, \lambda=1.5406 \AA$ ) were measured with a Rigaku D/MAX2400 . Elemental analyses of the products were conducted by the KEVEX energy-dispersive X-ray spectroscopy (EDS). Hitachi H-600 transmission electron microscopy (TEM) and Hitachi JSM-6701F field emission scanning electron micrograph (SEM) were taken to examine the morphology and dimension of the cobalt-iron PBAs nanotubes.

\section{Results and Discussion}

Morphology. The TEM micrographs of compounds 1, 2 and $\mathbf{3}$ were shown in Fig. 1. and the SEM image of compound $\mathbf{1}$ was shown in Fig. 2. The results indicate that the morphology of the three samples exhibits similar nanotube structure. The average diameters of the nanotubes are $\sim 1 \mu \mathrm{m}, \sim 1 \mu \mathrm{m}$ and $\sim 1$ $\mu \mathrm{m}$, respectively, and the wall thickness are all about $250 \mathrm{~nm}$, while the length of the nanotubes goes up to $4 \sim 15 \mu \mathrm{m}$, and a majority of them are open-ended. Diffuse rings in the selectedarea electron diffraction (SAED) pattern of the compound $\mathbf{1}$ nanotubes (Fig. 3) imply that it was polycrystalline. Similar

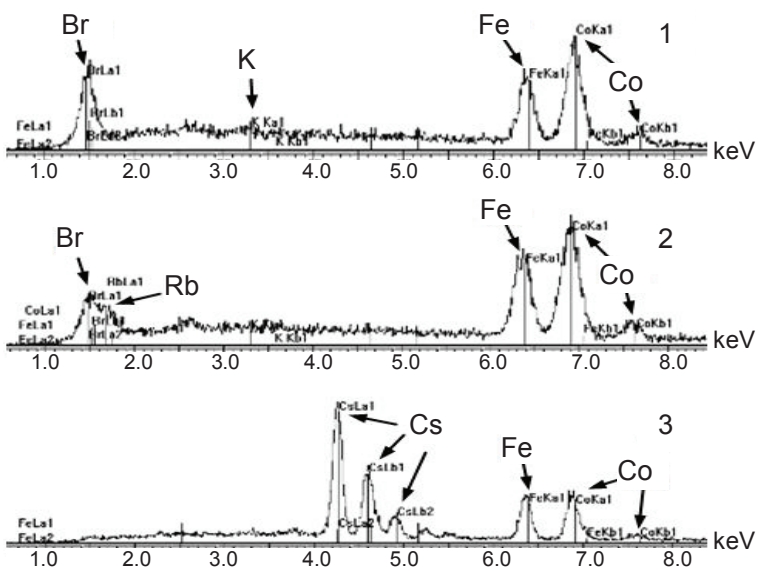

Figure 4. EDS pattern of PBAs nanotubes.

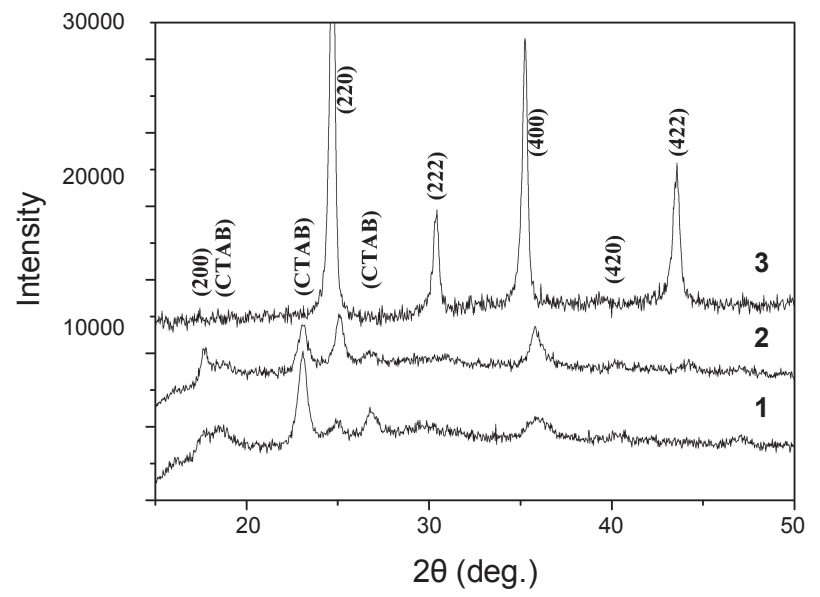

Figure 5. XRD pattern of PBAs nanotubes.

SAED patterns are observed for compounds 2, 3 as well and same results are obtained, both polycrystallines.

EDS and XRD analysis. To confirm the composition of the prepared compounds, EDS pattern of the compounds was performed to identify the elements present (Fig. 4). The EDS pattern of the compound 1 confirmed the existence of $\mathrm{K}, \mathrm{Fe}$, $\mathrm{Co}$ and $\mathrm{Br}$ in the products derived. The presence of $\mathrm{Rb}, \mathrm{Fe}, \mathrm{Co}$ and $\mathrm{Br}$ are verified for compound $\mathbf{2}$, but compound $\mathbf{3}$ was found to consist of only $\mathrm{Cs}, \mathrm{Fe}$ and $\mathrm{Co}$, without $\mathrm{Br}$ element. The presence of $\mathrm{Br}$ in compounds $\mathbf{1}$ and $\mathbf{2}$ indicated that a certain amount of surfactants are contained in them, while compound $\mathbf{3}$ is pure PBAs. Hence, the formula of the three samples may be derived as $\mathrm{K}_{0.03} \mathrm{Co}_{1.80}\left[\mathrm{Fe}(\mathrm{CN})_{6}\right]$.nCTAB, $\mathrm{Rb}_{0.19} \mathrm{Co}_{1.44}$ $\left[\mathrm{Fe}(\mathrm{CN})_{6}\right] \cdot n \mathrm{CTAB}, \mathrm{Cs}_{1.56} \mathrm{Co}_{1.07}\left[\mathrm{Fe}(\mathrm{CN})_{6}\right]$, respectively. We speculated that the $\mathrm{CTA}^{+}$can combine with the negatively charged PBAs in $\mathbf{1}$ and $\mathbf{2}$ to form complexes via electrostatic interactions. As to compound $\mathbf{3}$, however, the combination between $\mathrm{CTA}^{+}$and PBAs barely exists because the content of alkali cation $\mathrm{Cs}^{+}$is much higher and the steric hindrance effect of $\mathrm{CTA}^{+}$is greater than $\mathrm{Cs}^{+}$. And compared with $\mathrm{K}^{+}$(compound 1) and $\mathrm{Rb}^{+}$(compound 2), more $\mathrm{Cs}^{+}$(compound 3) had entered into the tetrahedral sites because of the smallest hydrated ionic radius. As a result, no $\mathrm{Br}$ element is observed in compound 3 . 
The powder diffraction pattern of the compounds was shown in Fig. 5. The characteristic peaks shown in the spectrum reveal that the powders have a face-centered cubic (FCC) structure (JCPD card No.75-0038). ${ }^{18,19}$ There are some peaks of CTAB in the XRD pattern of compounds $\mathbf{1}$ and $\mathbf{2}$, which are in agreement with the EDS results. In the compound $\mathbf{3}$, the $\mathrm{Fe} / \mathrm{Co}$ ratio is higher, so the (111) reflection is completely suppressed and disappeared. ${ }^{20}$ Moreover, because a quarter of the tetrahedral interstitial sites is filled with cesium atoms, the metal atoms and the alkali cations should scatter in phase for the (220) and (400) reflections and out of phase for the (200) and (420) reflections. ${ }^{20}$

Formation mechanism. CTAB has been systematically studied in the synthesis of mesostructured materials and may form spherical, cylindrical micelle, or even higher-order phases depending on the solution conditions. ${ }^{21}$ In our case, the concentration of the $\mathrm{CTAB}$ is above the critical micelle concentration $(\mathrm{CMC}),{ }^{22}$ and the shape of aggregates may be rodlike in order that the system finds its minimum energy configuration. ${ }^{23}$ The $\left[\mathrm{Fe}(\mathrm{CN})_{6}\right]^{3-}$ anions present in the reaction mixture tend to electrostatically interact with the surfactant cationic head groups, $\mathrm{CTA}^{+}$, to form $\mathrm{CTA}^{+}-\left[\mathrm{Fe}(\mathrm{CN})_{6}\right]^{3-}$ ion pairs. Therefore, the electrostatic interactions are the cause of inorganic-surfactant composites under this reaction condition. When the $\mathrm{Co}^{2+}$ solution was added, the $\mathrm{Co}^{2+}$ reacted with the ion pair to form a $\mathrm{CTA}^{+}-$ $\left[\mathrm{Fe}(\mathrm{CN})_{6}\right]^{3-}-\mathrm{Co}^{2+}$ in the surface of the rodlike micelle. Thus, after the CTAB soft template was removed, the PBAs nanotubes were obtained.

\section{Conclusions}

In summary, three Cobalt-iron PBAs nanotubes, $\mathrm{K}_{0.03} \mathrm{Co}_{1.80}$ $\left[\mathrm{Fe}(\mathrm{CN})_{6}\right] \cdot \mathrm{nCTAB}, \mathrm{Rb}_{0.19} \mathrm{Co}_{1.44}\left[\mathrm{Fe}(\mathrm{CN})_{6}\right] \cdot \mathrm{nCTAB}$, and $\mathrm{Cs}_{1.56}$ $\mathrm{Co}_{1.07}\left[\mathrm{Fe}(\mathrm{CN})_{6}\right]$ were prepared by CTAB soft template method. They are polycrystalline with FCC structures, with diameters of $\sim 1 \mu \mathrm{m}$ and lengths of $4 \sim 15 \mu \mathrm{m}$. The ion-exchange properties, battery applications, and magnetic properties of the formed Prussian blue nanotubes are to be investigated in the future. And the PBAs nanotubes inserted with metal possessed of catalytic properties may also be promising candidates for catalytic field. It should be noted that this method could be possibly applied to obtain the nanotubes of other Prussian blue analogues.

Acknowledgments. This work was supported by the Natio- nal Natural Science Foundation of China (Projects 20473035 and 20603014) and Committee of Science and Technology of Shanghai (Projects 0652nm010 and 08jc1408100).

\section{Reference}

1. Vaucher, S.; Fielden, J.; Li, M.; Dujardin, E.; Mann, S. Nano. Lett. 2002, 2, 225.

2. Mallah, T.; Thiebaut, S.; Verdaguer, M.; Veillet, P. Science 1993, 262,1554

3. Buser, H. J.; Schwarzenbach, D.; Petter, W. Inorg. Chem. 1977, 16, 2704.

4. Zhou, P. H.; Xue, D. S.; Luo, H. Q.; Chen, X. G. Nano. Lett. 2002 , 2, 845 .

5. Yamada, M.; Arai, M.; Kurihara, M.; Sakamoto, M.; Miyake, M. J. Am. Chem. Soc. 2004, 126, 9482.

6. Clavel, G.; Larionova, J.; Guari, Y.; Guerin, C. Chem. Eur. J. 2006, 12, 3798.

7. Clavel, G.; Guari, Y.; Larionova, J.; Guerin, C. New. J. Chem. 2005, 29, 275.

8. Bagkar, N.; Ganguly, R.; Choudhury, S.; Hassan, P. A.; Sawanta, S.; Yakhmi, J. V. J. Mater. Chem. 2004, 14, 1430.

9. Dominguez-Vera, J. M.; Colacio, E. Inorg. Chem. 2003, 42, 6983.

10. Uemura, T.; Kitagawa, S. J. Am. Chem. Soc. 2003, 125, 7814.

11. Moore, J. G.; Lochner, E. J.; Ramsey, C.; Dalal, N. S.; Stiegman, A. E. Angew. Chem. Int. Ed. 2003, 42, 2741

12. Vo, V.; Van, M. N.; Lee, H. I.; Kim, J. M.; Kim, Y.; Kim, S. J. Mater. Chem. Phys. 2008, 107, 6.

13. Fornasieri, G.; Bleuzen, A. Angew. Chem. Int. Ed. 2008, 47, 7750.

14. Shi, Y. M.; Zhou, B.; Wu, P. J. Electroanal. Chem. 2007, 611, 1.

15. Fang, J.; Du, X. L.; Zhao, J. H.; Shen, W. G. Chinese. J. Inorg. Chem. 2007, 23, 923.

16. Fang, J.; Du, X. L.; Zhao, J. H.; Shen, W. G. Acta Chim. Sinica. 2007, 65, 2639.

17. Johansson, A.; Widenkvist, E.; Lu, J.; Boman, M.; Jansson, U. Nano. Lett. 2005, 5, 1603.

18. Choudhury, S.; Dey, G. K.; Yakhmi, J. V.J. Cryst. Growth. 2003, 258, 197.

19. Bleuzen, A.; Lomenech, C.; Escax, V.; Villain, F.; Varret, F.; Cartier dit Moulin, C.; Verdaguer, M. J. Am. Chem. Soc. 2000, 122, 6648.

20. Cartier dit Moulin, C.; Villain, F.; Bleuzen, A.; Arrio, M. A.; Sainctavit, P.; Lomenech, C.; Escax, V.; Baudelet, F.; Dartyge, E.; Gallet, J. J.; Verdaguer, M. J. Am. Chem. Soc. 2000, 122, 6653.

21. Fendler, J. H.; Fendler, E. J. Catalysis in Micellar and Macromolecular Systems; Academic Press: New York, U. S. A., 1975.

22. Li, W.; Han, Y. C.; Zhang, J. Z.; Wang, B. G. Colloid J. 2005, 67, 159.

23. Biz, S.; Occelli, M. L. Catal. Rev. Sci. Eng. 1998, 40, 329. 